\title{
BMJ Open Comparison of efficacy and safety of His-Purkinje system pacing versus cardiac resynchronisation therapy in patients with pacing-induced cardiomyopathy: protocol for a randomised controlled trial
}

Junjun Chen, ${ }^{1}$ Liting Cheng, ${ }^{2}$ Zefeng Wang, ${ }^{2}$ Zhuo Liang, ${ }^{2}$ Ruiqing Dong, ${ }^{1}$ Fei Hang, ${ }^{1}$ Jieruo Chen, ${ }^{2}$ Xinlu Wang, ${ }^{1}$ Ziyu Wang, ${ }^{1}$ Xiao Du (D),${ }^{1}$ Junmeng Zhang, ${ }^{1}$ Yongquan $\mathrm{Wu}$ (1) ${ }^{2}$

To cite: Chen J, Cheng L, Wang Z, et al. Comparison of efficacy and safety of HisPurkinje system pacing versus cardiac resynchronisation therapy in patients with pacinginduced cardiomyopathy: protocol for a randomised controlled trial. BMJ Open 2021;11:e045302. doi:10.1136/ bmjopen-2020-045302

- Prepublication history for this paper is available online. To view these files, please visit the journal online (http://dx.doi org/10.1136/bmjopen-2020045302).

$\mathrm{JC}, \mathrm{LC}, \mathrm{JZ}$ and $\mathrm{YW}$ contributed equally.

Received 01 October 2020 Accepted 15 July 2021

Check for updates

(c) Author(s) (or their employer(s)) 2021. Re-use permitted under CC BY-NC. No commercial re-use. See rights and permissions. Published by BMJ.

${ }^{1}$ Department of Cardiology, Beijing An Zhen Hospital, Chaoyang-qu, Beijing, China ${ }^{2}$ Beijing An Zhen Hospital, Chaoyang-qu, China

Correspondence to Dr Yongquan Wu; wuyongquan67@163.com

\section{ABSTRACT}

Introduction Recent studies have shown that the His-Purkinje system pacing (HPSP) can achieve electrocardiomechanical synchronisation, and thus improve cardiac function. For patients with pacinginduced cardiomyopathy (PICM) who should be treated with pacemaker upgrade, the HPSP is a viable alternative to cardiac resynchronisation therapy (CRT). However, no randomised controlled trial has been performed to evaluate the efficacy and safety of HPSP in patients with PICM. The present study compared the efficacy and safety of HPSP with that of traditional CRT in the treatment of patients with PICM.

Methods and analysis This study is a single-centre, randomised controlled non-inferiority trial. This trial was carried out at the cardiac centre of Beijing Anzhen Hospital. A total of 46 patients with PICM who needed pacemaker upgrade treatment between January 2022 and December 2023 will be enrolled in this study. Patients will be randomised into an investigational group (HPSP) and a control group (CRT) at a 1:1 ratio. The primary outcome is the duration of $Q R S$ complex (QRS width), and the secondary outcomes are NT-proBNP (N terminal pro B type natriuretic peptide), $C$ reactive protein, the number of antibiotics used, left ventricular ejection fraction, end systolic volume, end diastolic volume, the hospitalisation duration, the incidence of postoperative infection, pacemaker parameters (threshold, sensing and impedance), the 6-minute walking test, and quality of life (36-Item Short Form Survey scale), all-cause mortality, cardiovascular death, heart failure-related rehospitalisation rate, other rehospitalisation rates, major complication rates and procedure costs.

Ethics and dissemination This study has been approved by the Beijing Anzhen Hospital Medical Ethics Committee (No. 2020043X).

Trial registration number Chinese Clinical Trial Registry (ChiCTR2000034265).
Strengths and limitations of this study

- A randomisation sequence will be generated by a centralised computer system and a specific investigator will be assigned to this job.

- Personnel will undergo training before this trial.

- A risk assessment team will evaluate the entire study.

- Three specific non-investigators will perform the data entry with EpiData.

- Five experienced surgeons will perform the operations in turn.

- The doctors who will evaluate the ventricular function will not be blinded, since they will be able to see where the ventricular lead is positioned.

\section{INTRODUCTION}

Hyman $e t a l^{1}$ first proposed the concept of a 'pacemaker' in 1932. At present, pacemaker implantation has become the first-line treatment for patients with bradycardia. Right ventricular apex pacing (RVAP) remains the first choice for many cardiologists because of the simple technique of implanting electrodes into the right ventricular apex. However, RVAP can cause biventricular and left ventricular conduction asynchrony and ventricular remodelling, ${ }^{2}$ and lead to pacing-induced cardiomyopathy (PICM) in about $10 \%-20 \%$ of patients. PICM is associated with deterioration of cardiac function, increased mortality and rehospitalisation rates. ${ }^{3}$ Although pacemaker programmed control can reduce unnecessary right ventricular pacing, it still cannot improve patient clinical prognosis.

At present, traditional cardiac resynchronisation therapy (CRT) is the first choice for 
pacing upgrade in patients with PICM. Previous studies have shown that patients with PICM following CRT had improved cardiac function, reduced ventricular remodelling and decreased readmission rate. ${ }^{4}$ In addition, CRT achieves a similar cardiac contraction pattern by synchronously pacing the left and right ventricles, however, CRT is still not physiological pacing. Some patients experience complications such as failure of left ventricular lead implantation, electrode dislocation, phrenic nerve stimulation and no response to CRT. ${ }^{5}$

In 1970, Narula et al proposed the feasibility of achieving physiological ventricular synchronous activation by pacing the His bundle. ${ }^{6}$ In 2000, Deshmukh et al successfully performed atrioventricular node ablation in patients with atrial fibrillation with impaired left ventricular systolic function for the first time using His bundle pacing. ${ }^{7}$ The cardiac structure and function of the patients treated with His bundle pacing were significantly improved. ${ }^{7}$ Currently, His-Purkinje system pacing (HPSP), which includes His bundle pacing (HBP) and left bundle branch area pacing (LBBaP), is considered to be the most physiological pacing mode. ${ }^{8}$ Studies have shown that for patients with cardiac insufficiency who need CRT, HBP can deliver greater improvement in cardiac electrical synchronisation than traditional CRT, and may further improve cardiac function. ${ }^{9} \mathrm{LBBaP}$ was developed based on HBP. Since LBBaP was reported by Huang $e a^{10}$ in 2017, it has attracted wide attention in the field of cardiac pacing. In 2017, Huang et al first reported the clinical application of LBBaP in a patient with heart failure complicated with left bundle branch block. After the 1-year follow-up, the pacing parameters were stable and no complications were found. ${ }^{10}$ Compared with HBP, LBBaP is relatively simple and has a higher success rate. Several studies, including those from our centre, have shown that LBBaP can also achieve cardiac electromechanical synchronisation and improve cardiac function. ${ }^{11}$ In an early clinical trial performed at our centre, five patients with PICM requiring pacemaker upgrade were treated with HPSP. These patients had improved left ventricular ejection fraction (LVEF) and narrower paced QRS waves compared with HBP. However, no randomised controlled study has evaluated the efficacy and safety of HPSP in patients with PICM. Therefore, on the basis of previous studies, we aim to compare the efficacy and safety of HPSP with that of traditional CRT in the treatment of patients with PICM.

\section{Research questions and study hypothesis Objective}

For patients with PICM who need upgrade treatment, there is limited guidance regarding choice of CRT or HPSP. This study will explore whether HPSP is noninferior to CRT in the following aspects: (1) QRS width, (2) NT-proBNP, (3) C reactive protein (CRP), (4) LVEF, (5) left ventricular end systolic volume, (6) left ventricular end diastolic volume, (7) the hospitalisation duration, (8) the number of antibiotics used, (9) the incidence
From January 2022 to December 2023, eligible patients with PICM who need pacemaker upgrade treatment

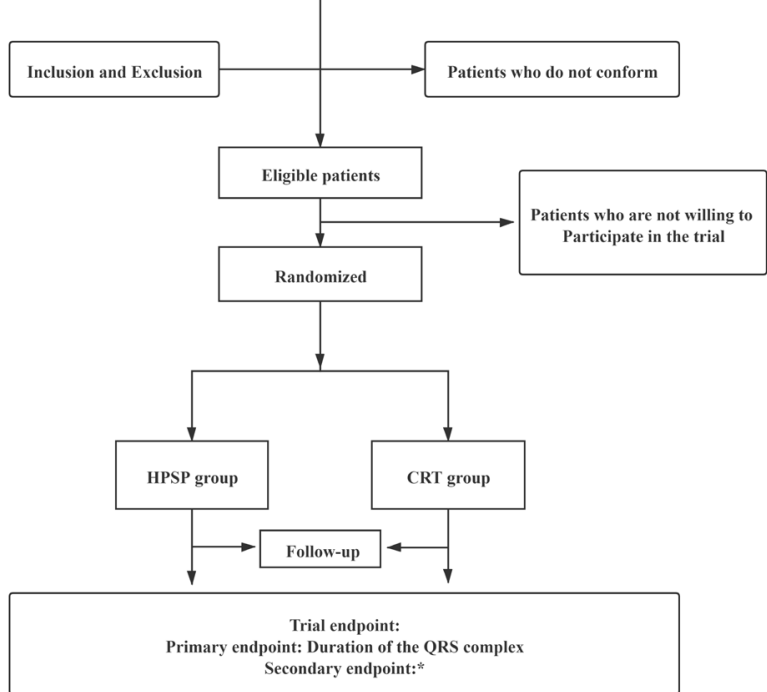

Figure 1 Efficacy index: *(a) short-term: NT-proBNP, LVEF, end systolic volume, end diastolic volume, the 6-minute walking test, quality of life (SF-36 scale) and NYHA classification; (b) long-term: pacemaker parameters (threshold, sensing and impedance), all-cause mortality, cardiovascular death, rehospitalisation rate of heart failure and other rehospitalisation rates. Safety index: CRP; the use of antibiotics; incidence of infection after reimplantation; major complication rates: cardiac perforation, haemopericardium, malignant arrhythmias, sudden cardiac death and acute myocardial infarction. Health economic index: procedural costs (ie, pacemaker, leads, etc) and hospitalisation duration. CRP, C reactive protein; CRT, cardiac resynchronisation therapy; HPSP, His-Purkinje system pacing; LVEF, left ventricular ejection fraction; NYHA, New York Heart Association; PICM, pacing-induced cardiomyopathy; SF-36, 36-Item Short Form Survey.

of postoperative infection, (10) pacemaker parameters: threshold, sensing and impedance, (11) 6-minute walking test score, (12) 36-Item Short Form Survey (SF-36) scale, (13) all-cause mortality, (14) cardiovascular death, (15) the heart failure-related rehospitalisation rate, (16) other rehospitalisation rates, (17) major complication rates, and (18) New York Heart Association (NYHA) functional classification.

\section{Hypothesis}

We hypothesised that the QRS width of HPSP is noninferior to that of CRT in patients with PICM.

\section{METHODS AND ANALYSIS \\ Study design}

A single-centre, randomised controlled non-inferiority trial will be conducted in the Heart Center of Beijing Anzhen Hospital. Eligible patients with PICM who need pacemaker upgrade treatment between January 2022 and December 2023 were enrolled in this study (figure 1).

This study has been approved by the Beijing Anzhen Hospital Medical Ethics Committee ((No. 2020043X). 
Participants evaluated in this trial will sign an informed consent form in which they will agree to participate in the trial while they are in the hospital as well as during follow-up.

\section{Subjects}

\section{Inclusion criteria}

Patients who have all of the following 1-4 items and any one of the 5-6 items will be included in this study:

1. Implantation with single-chamber/dual-chamber pacemakers/implanted cardioverter defibrillator with right ventricular electrode implanted in the right ventricular apex or low septum.

2. Age between 18 and 80 years.

3. Longer than 3-month medication.

4. Expected survival time $>1$ year.

5. A ventricular pacing proportion $>40 \%$ and an LVEF $<50 \%$.

6. If baseline LVEF was $\geq 50 \%$, LVEF $\leq 40 \%$ after RVAP treatment; and for patients with an LVEF $<50 \%$, after RVAP treatment, the decrease was $\geq 10 \%$ compared with before treatment. ${ }^{3}$

\section{Exclusion criteria}

Patients who have one of the following were excluded from this trial:

1. Inability to cooperate with follow-up.

2. An estimated glomerular filtration rate $<30 \mathrm{~mL} / \mathrm{min}$.

3. Septic shock.

4. Advanced malignant tumour.

5. Pregnancy or prepared to get pregnant.

6. Cardiac tamponade or major hydropericardium.

7. Atrial fibrillation.

\section{Study grouping and intervention}

1. CRT group: the CRT group is defined as follows: (1) complete CRT treatment or (2) failure to be treated with CRT or eventually treated with HPSP. Failure to be treated with CRT is defined as follows: (a) failure to place the left ventricular lead in patients with coronary artery variation and/or coronary vein stenoses; (b) diaphragm stimulus occurred at multiple points with the left ventricular electrode; or (c) the left ventricular electrode pacing threshold was greater than 3 $\mathrm{V}$ at $0.4 \mathrm{~ms}$.

For intervention, the subclavian vein or axillary vein of patients will be punctured for venous access and the implanted left ventricular lead will pass the vein into the appropriate location of the cardiac cavity, and the parameters (perception, threshold, impedance) of the test wire will be recorded.

2. HPSP group: the HPSP group is defined as follows: (3) complete HPSP treatment or (4) failure to be treated with HPSP or eventually treated by CRT. Failure to be treated with HPSP is defined as follows: (a) left bundle branch block could not be corrected; (b) stiffness of the ventricular septum made it difficult to implant the ventricular lead; or (c) the ventricular lead pacing threshold was greater than $3 \mathrm{~V}$ at $0.4 \mathrm{~ms}$. The intentionto-treat set is used to compare $(1)+(2)$ with $(3)+(4)$. The per-protocol analysis set is used to compare (1) with (3).

3. HPSP group: for intervention, the HPSP lead will be implanted using the 'New Nine Partition Method', as previously detailed. ${ }^{12}$ The surgical procedures of the patients in the HPSP group include HBP and LBBaP, which will be decided by the operators according to the situation during the operation. The 7-Fr and 8-Fr introducer sheaths (Medtronic, Minneapolis, Minnesota, USA) will be inserted through the guidewires after venous puncture. The C315 sheath (Medtronic, Minneapolis, Minnesota, USA) will be inserted through 8Fr sheath. The ventricular pacing lead (model 3830, Medtronic, Minneapolis, Minnesota, USA) will then be implanted through C315 sheath. As for RA (Right Atrium), 4574 or 5076 electrode (Medtronic, Minneapolis, Minnesota, USA) will be implanted through a 7-Fr sheath. The threshold will be tested during the procedure using a Medtronic pacemaker programmer.

4. CRT group: subclavican or axillary venous puncture will be performed three times. Two 7-Fr and one 9-Fr sheaths (Medtronic, Minneapolis, Minnesota, USA) will then be implanted. For left ventricular electrode, 6250 and 6248 sheaths (Medtronic Minneapolis, Minnesota, USA) will be inserted through 9-Fr sheath. After then, a 4196/4296/4396/4298/4195 electrode (Medtronic, Minneapolis, Minnesota, USA) will then be implanted. As for right ventricle, a 4074 or 5076 electrode (Medtronic, Minneapolis, Minnesota, USA) will be inserted through 7-Fr sheath. In the end, a 4574 or 5076 electrode will be inserted into RA through 7-Fr sheath.

\section{Randomisation and masking}

A non-investigator will be assigned to generate a randomisation sequence using a centralised computer system. Subjects who are selected based on the enrolment criteria will be assigned to either CRT or HPSP groups at a 1:1 ratio. Patients' medical information will be kept confidential.

\section{Study procedures \\ Screening}

Patients with PICM will be treated at the Heart Center of Beijing Anzhen Hospital between January 2022 and December 2023 and screened according to study enrolment criteria. Participants evaluated in this trial will sign an informed consent form in which they will agree to participate in the trial. Echocardiography and electrogram will be evaluated by two experienced doctors. The QRS complex duration and LVEF are essential parameters for evaluation of heart function and cardiac mechanical synchrony. A venous phase coronary angiogram in left anterior oblique and right anterior oblique will be used to assess eligibility of the venous system in meeting study enrolment criteria. 
Table 1 Follow-up-related content

\begin{tabular}{|c|c|c|c|c|}
\hline & $\begin{array}{l}\text { Group screening } \\
\text { period }\end{array}$ & $\begin{array}{l}\text { One month } \\
\text { after operation }\end{array}$ & $\begin{array}{l}\text { Three months } \\
\text { after operation }\end{array}$ & $\begin{array}{l}\text { Six months } \\
\text { after operation }\end{array}$ \\
\hline Informed consent & $\sqrt{ }$ & & & \\
\hline CRP & $\sqrt{ }$ & $\sqrt{ }$ & $\sqrt{ }$ & $\sqrt{ }$ \\
\hline Routine blood test & $\sqrt{ }$ & & & \\
\hline ECG & $\sqrt{ }$ & $\sqrt{ }$ & $\sqrt{ }$ & $\sqrt{ }$ \\
\hline Ultrasonic cardiogram (especially LVEF) & $\sqrt{ }$ & $\sqrt{ }$ & $\sqrt{ }$ & $\sqrt{ }$ \\
\hline X-ray examination & $\sqrt{ }$ & & & \\
\hline Incidence of infection after reimplantation & & $\sqrt{ }$ & $\sqrt{ }$ & $\sqrt{ }$ \\
\hline $\begin{array}{l}\text { Pacemaker programmed control (threshold, sensing } \\
\text { and impedance) }\end{array}$ & & $\sqrt{ }$ & $\sqrt{ }$ & $\sqrt{ }$ \\
\hline Hospitalisation duration & & $\sqrt{ }$ & $\sqrt{ }$ & $\sqrt{ }$ \\
\hline $\begin{array}{l}\text { General condition of patients, rehospitalisation, } \\
\text { survival and other information }\end{array}$ & & $\sqrt{ }$ & $\sqrt{ }$ & $\sqrt{ }$ \\
\hline
\end{tabular}

CRP, C reactive protein; LVEF, left ventricular ejection fraction; 6MWT, 6-minute walking test; NYHA, New York Heart Association; SF-36, 36Item Short Form Survey.

\section{Data collection}

Demographic and baseline clinical characteristics of all participants, including age, sex, body mass index, history (ie, cardiomyopathy, coronary heart disease, hypertension and diabetes mellitus), medication history and blood sample test results, were gathered using case report forms (CRFs).

During the procedure, intraoperative data collection forms will be used to collect the following information: complications, X-ray duration, the radiation dosage, and detailed information on the pacemaker and pacing lead, pacemaker parameters (voltage, sensing, impedance) and QRS duration.

\section{Randomisation and follow-up}

Randomisation will be performed between January 2022 and December 2023 with a 6-month follow-up. Outpatients will be followed up at 1, 3 and 6 months postoperation. Routine blood tests, echocardiography (especially LVEF) and electrograms will be performed during the follow-up period. Specifically, the duration of QRS complex will be evaluated. With or without postoperative infection, the number of antibiotics used, rehospitalisation rate, hospitalisation duration, pacemaker parameters (threshold, sensing and impedance) will be recorded (table 1).

\section{Risk management}

A risk assessment team will evaluate the entire study for all mortality and morbidity events. Patients who develop any kind of cardiovascular disease will be admitted and will have a full physical examination.

\section{Outcomes}

Primary outcome

Duration of the QRS complex will be evaluated at 1,3 and 6 months postoperation by two experienced doctors (recorded by $25 \mathrm{~mm} / \mathrm{s}$ and $100 \mathrm{~mm} / \mathrm{s}$, respectively).

\section{Secondary outcomes}

\section{Efficacy outcomes}

Short-term outcomes: NT-proBNP, LVEF, end systolic volume, end diastolic volume, the 6-minute walking test, quality of life (SF-36 scale) and NYHA functional classification; and long-term outcomes: pacemaker parameters (threshold, sensing and impedance), all- cause mortality, cardiovascular death, heart failure-related rehospitalisation rate and other rehospitalisation rates.

\section{Safety outcomes}

CRP; the use of antibiotics; incidence of infection after reimplantation; major complication rates: cardiac perforation, haemopericardium, malignant arrhythmias, sudden cardiac death and acute myocardial infarction.

\section{Health economic outcomes}

Procedural costs (ie, pacemaker, leads) and hospitalisation duration. 


\section{Data management and monitoring}

\section{Before the trial}

Personnel will undergo training prior to the initiation of this trial. Random team groupings will be performed. The operation team will uphold all technical standards. The echocardiography measurement team will perform the echocardiography measurements. The data processing team will use EpiData to perform the training and standardise the input using a CRF table. Crossover will not be allowed among the randomly divided teams, including the operation and data entry teams. All personnel will sign a patient privacy confidentiality agreement.

\section{During the trial}

Patients will be randomly divided into two groups. The patients' crossover rate will be controlled to be less than $10 \%$. Five operators, who have performed at least 50 CRT and 50 HPSP, will be selected in this study and randomised to perform the surgical treatment. Three adjudicators will evaluate the relevant indicators, with two personnel collecting and collating the data and one person checking the data. No crossover will be allowed between data collectors and the operators.

\section{Interim analyses}

An interim analysis will be conducted in July 2022 to evaluate the complication rate and early signal for inferiority in HPSP. If the complication rate is higher than $10 \%$, the trial will be stopped early. The crossover rate between the two groups will also be evaluated, which is generally no more than $5 \%$.

\section{After the trial}

Research documents will be collected and stored.

\section{Sample size calculation}

This study is designed as a non-inferiority trial to evaluate the efficacy and safety of HPSP in the treatment of patients with PICM. The primary outcome is the duration of the QRS complex. According to our previous results in five patients with PICM submitted to upgrade with HPSP, we showed a further narrowing of the QRS, in comparison with CRT, of $11 \mathrm{~ms}$ after 3 months. The SD in the duration of the QRS complex in the HPSP group was 11, and the SD in the duration of QRS complex in the CRT group was 14. The non-inferiority margin was $1 / 5$ (2.2 $\mathrm{ms}$ ) of the difference between the two groups, which means that HPSP is not inferior to CRT. Pass 11.0 (non-inferiority test) was used to compare the sample size differences between the two groups. The significance level (a) was 0.025 , and the assurance level (1- $\beta$ ) was 0.90 . At least 21 cases need to be enrolled in each of the two groups, with a total of 42 cases. Loss to follow-up is considered less than $10 \%$. In the present study, a total of 46 cases will be enrolled.

\section{Statistical analysis}

Three non-investigators will perform the data entry with EpiData. Two independent statisticians will perform the statistical analysis. Data will be analysed with SPSS statistics (IBM, V.23). Normally distributed continuous variables will be expressed as the mean $\pm \mathrm{SD}$ and non-uniformly distributed data will be expressed as the median (Q1 and Q3). Comparison of means (history, medication history) between groups will be analysed by the independent samples t-test for normally distributed data, and the Mann-Whitney U test for non-uniformly distributed data. A Kaplan-Meier curve will be used to determine the event rate between the two groups over time, and the log rank test will be used to compare two groups. The Cox proportional risk model will be used to calculate the HR. A p value of $<0.05$ will be considered statistically significant.

\section{Planned subgroup analyses}

1. Sex.

2. Ejection fraction.

3. LVEF.

4. Six-minute walking test.

5. NYHA classification.

6. Quality of life (SF-36 scale).

\section{Bias}

Patients will be consecutively enrolled from the cardiac centre of Beijing Anzhen Hospital. A total of 46 patients with PICM requiring pacemaker upgrade therapy will be enrolled. Using a centralised computer system, participants will be assigned to either the CRT or HPSP group at a $1: 1$ ratio.

\section{Patient and public involvement}

Patients or the general public will not be involved in the design, conduct, reporting or dissemination of the research.

\section{Ethics and safety considerations}

This study has been approved by the Beijing Anzhen Hospital Medical Ethics Committee (No. 2020043X) and abides by the principles of the Helsinki Declaration. Participants to be evaluated in this trial will provide informed consent and sign an involvement statement in which they will agree to participate in the trial while they are in the hospital as well as during follow-up. All research documents will be collected and stored at Beijing Anzhen Hospital.

\section{Implementation and dissemination}

This single-centre, randomised controlled non-inferiority trial will be conducted at the cardiac centre of Beijing Anzhen Hospital between January 2022 and December 2023. After this trial, the results of this study will be shown at domestic and international conferences for research and further study.

\section{DISCUSSION}

PICM is a severe complication triggered by the longterm presence of implanted pacemakers and may lead to heart failure and end-stage heart disease if not treated 
appropriately and timely. Although CRT currently remains the mainstay treatment of PICM, its shortcomings have been noted for a long time. For example, CRT is not a physiological pacing technique; it initiates the pacing from the apex of the right ventricle and from the free wall of the left ventricle. ${ }^{13}{ }^{14}$ Also, a subset of patients with heart failure had little or no response to CRT. ${ }^{15}$ In recent years, HPSP has emerged as a viable alternative to CRT to treat patients with PICM. For instance, HBP, one of two HPSP, has been shown to successfully narrow QRS duration, and improve LVEF and NYHA functional classification in 95 of 106 patients examined. ${ }^{16}$ The HisSYNC trial has also shown that HBP results in a narrower QRS duration compared with CRT, while both of them achieved comparable amelioration with regard to cardiac function, including LVEF, 6-minute walking test, NYHA functional classification and quality of life. ${ }^{17}$ These studies together support the premise that HBP may serve as a viable alternative to CRT in patients with heart failure. While HBP has been previously studied for heart failure treatment, few studies have been performed on LBBaP, the other HPSP, in terms of its treatment in patients with PICM. Our previous work improved the LBBaP implantation scheme and put forward the 'New Nine Partition Method' for LBBaP implantation, which is safe, efficient and technically simple. ${ }^{118}$ Our centre has also completed a comparative study of LBBaP and RVAP in patients with atrioventricular block with normal cardiac function. Recent follow-up has shown that LBBaP can improve left ventricular synchronisation and cardiac function, further confirming that the 'New Nine Partition Method' LBBaP is safe and effective. ${ }^{11}$ However, thus far, no clinical trial has been performed to evaluate the application of HPSP and traditional CRT in patients with PICM with pacemaker upgrade.

Based on the findings from our group and others, we proposed this clinical trial to explore the efficacy and safety of HPSP and compare outcomes with that of traditional CRT in patients with PICM pacemaker upgrade.

In this proposed trial, QRS duration will be used as the primary outcome. In a 15-year multicentre retrospective study performed in Korea, a prolonged QRS duration was observed in patients with PICM. ${ }^{19}$ Other studies have also shown that wider or prolonged QRS duration indicates high risk of development of PICM, ${ }^{20}$ and CRT efficiently reversed abnormal QRS duration in patients with PICM. Moreover, a wide QRS complex is currently one of major indicators for patients with heart failure to be selected for CRT. ${ }^{21}$ Hence, it is well appreciated that the abnormal QRS duration is closely linked to PICM, and that the QRS duration from abnormality to normality indicates the clinical improvement of patients with PICM. Therefore, QRS duration is an appropriate primary outcome measure for this clinical trial.

To further strengthen the significance of this trial, a number of secondary outcome parameters will also be captured. These parameters, such as NT-proBNP, LVEF and NYHA functional classification, are well-known and recognised indicators of cardiac injury and function. For instance, the circulating levels of NT-proBNP has been extensively used as a reliable biomarker for the severity of cardiac inflammation in the clinic. ${ }^{22}$ Further, NYHA functional classification has been widely used to evaluate the cardiac function of patients with heart failure before and after treatment. ${ }^{23}$ Other parameters, such as acute myocardial infarction and malignant arrhythmia, represent the adverse outcomes potentially associated with the treatment. By evaluating the primary and secondary outcomes proposed in this protocol, the findings from the proposed study will provide direct evidence whether the curative effect of HPSP is not inferior to that of traditional CRT in patients with PICM, given that HPSP is considered a more physiological pacing method than CRT.

As mentioned above, HPSP includes HBP and LBBaP. While both are considered physiological pacing methods, evidence suggests that $\mathrm{LBBaP}$ is associated with more advantages compared with HBP. A recent study showed that HBP had a lower success rate and higher pacing threshold than LBBaP in treatment of patients who needed transcatheter aortic valve replacement. ${ }^{24-26}$ In addition, HBP is technically difficult, readily injures the His bundle branch, and has a relatively high lead revision rate. ${ }^{18}$ However, whether there is any differences in safety and efficacy as well as complications between HBP and LBBaP in treatment of patients with PICM is unknown. Therefore, ideally, HBP and LBBaP should be examined separately and compared with regard to efficacy and safety in patients with PICM. However, the proposed design is not powered for this analysis. A future large cohort study is necessary to separately investigate and compare the efficacy and safety of HBP and LBBaP in the treatment of patients with PICM.

The following measures will be implemented to ensure objective findings from this trial: (1) patients will be strictly selected based on the inclusion and exclusion criteria, and randomly grouped; (2) all personnel will undergo training related to this trial; (3) surgeons who will perform HPSP are highly experienced; and (4) investigators who collect and analyse data will be blinded to the grouping of patients.

Some limitations of this prospective clinical trial should be noted. As mentioned above, the small sample size prevents us from separately investigating and comparing safety and efficacy between HBP and LBBaP. Also, the proposed trial is a single-centre trial. In addition, the proposed follow-up is relatively short, and long-term complications related to the respective treatment will not be investigated. In a future study, a longer follow-up will be needed to corroborate any findings obtained from the proposed trial.

In conclusion, we propose a prospective clinical trial, in which we will evaluate the efficacy and safety of HPSP treatment for patients with PICM and compare efficacy and safety of this treatment with CRT. With wellrecognised variables as primary and secondary outcomes, the proposed clinical trial will yield important evidence 
that may guide the clinical application of HPSP for patients with PICM.

Contributors JC and LC are joint first authors. Study design-JC, LC, ZW, ZL, RD, $\mathrm{FH}, \mathrm{JC}, \mathrm{XW}, \mathrm{ZW}, \mathrm{XD}, \mathrm{JZ}$ and YW. Drafting of the manuscript-JC and LC. Critical revision of the manuscript-JC, LC, ZW, JZ and YW. Approval of the final version for publication-all authors.

Funding This work was supported by the Beijing Hospitals Authority Clinical Medicine Development of Special Funding Support (grant no. XMLX202112).

Competing interests None declared.

Patient and public involvement Patients and/or the public were not involved in the design, or conduct, or reporting, or dissemination plans of this research.

Patient consent for publication Obtained.

Provenance and peer review Not commissioned; externally peer reviewed.

Open access This is an open access article distributed in accordance with the Creative Commons Attribution Non Commercial (CC BY-NC 4.0) license, which permits others to distribute, remix, adapt, build upon this work non-commercially, and license their derivative works on different terms, provided the original work is properly cited, appropriate credit is given, any changes made indicated, and the use is non-commercial. See: http://creativecommons.org/licenses/by-nc/4.0/.

\section{ORCID iDs}

Xiao Du http://orcid.org/0000-0002-3133-3532

Yongquan Wu http://orcid.org/0000-0001-9156-4669

\section{REFERENCES}

1 Albert S. Resuscitation of the stopped heart by intracardiac therapy: hyman. Arch Int Med 1930;46.

2 Khurshid S, Epstein AE, Verdino RJ, et al. Incidence and predictors of right ventricular pacing-induced cardiomyopathy. Heart Rhythm 2014;11:1619-25.

3 Merchant FM, Mittal S. Pacing induced cardiomyopathy. J Cardiovasc Electrophysiol 2020;31:286-92.

4 Shan P, Su L, Zhou X, et al. Beneficial effects of upgrading to his bundle pacing in chronically paced patients with left ventricular ejection fraction $<50$. Heart Rhythm 2018;15:405-12.

5 Zoppo F, Gagno G. Left ventricle automatic pacing threshold management in CRT systems: a comprehensive review. J Cardiovasc Electrophysiol 2020;31:2489-98.

6 Narula OS, Scherlag BJ, Samet P. Pervenous pacing of the specialized conducting system in man. His bundle and A-V nodal stimulation. Circulation 1970;41:77-87.

7 Deshmukh P, Casavant DA, Romanyshyn M, et al. Permanent, direct His-bundle pacing: a novel approach to cardiac pacing in patients with normal His-Purkinje activation. Circulation 2000;101:869-77.

8 Zhang J-M, Wang Z-F, JianZhang Y-QW. A new concept of resynchronization therapy for heart failure: cardiac resynchronization therapy by HPSP. Chinese $J$ Heart Failure Cardiomyopathy 2019;3:159-62.

9 Vijayaraman P, Herweg B, Ellenbogen KA, et al. His-optimized cardiac resynchronization therapy to maximize electrical resynchronization: a feasibility study. Circ Arrhythm Electrophysiol 2019;12:e006934.

10 Huang W, Su L, Wu S, et al. A novel pacing strategy with low and stable output: pacing the left bundle branch immediately beyond the conduction block. Can J Cardiol 2017;33:1731-6.

11 Zhang J, Wang Z, Cheng L, et al. Immediate clinical outcomes of left bundle branch area pacing vs conventional right ventricular pacing. Clin Cardiol 2019;42:768-73.

12 Zhang J, Wang Z, Zu L, et al. Simplifying physiological left bundle branch area pacing using a new nine-partition method. Can J Cardiol 2021;37:329-38.

13 Wu L, de Roest GJ, Hendriks ML, et al. The influence of right ventricular stimulation on acute response to cardiac resynchronisation therapy. Neth Heart J 2016;24:66-72.

14 Bilchick KC. Does cardiac resynchronization therapy benefit patients with right bundle branch block: left ventricular free wall pacing: seldom right for right bundle branch block. Circ Arrhythm Electrophysiol 2014;7:543-52.

15 European Society of Cardiology (ESC), European Heart Rhythm Association (EHRA), Brignole M, et al. 2013 ESC guidelines on cardiac pacing and cardiac resynchronization therapy: the task force on cardiac pacing and resynchronization therapy of the European Society of cardiology (ESC). developed in collaboration with the European heart rhythm association (EHRA). Europace 2013;15:1070-118.

16 Sharma PS, Dandamudi G, Herweg B, et al. Permanent hisbundle pacing as an alternative to biventricular pacing for cardiac resynchronization therapy: a multicenter experience. Heart Rhythm 2018:15:413-20.

17 Lustgarten DL, Crespo EM, Arkhipova-Jenkins I, et al. His-bundle pacing versus biventricular pacing in cardiac resynchronization therapy patients: a crossover design comparison. Heart Rhythm 2015;12:1548-57.

18 Cheng L-T, Zhang J-M, Wang Z-F, et al. Recent approaches to hispurkinje system pacing. Chin Med J 2019;132:190-6.

$19 \mathrm{Kim} \mathrm{JH}$, Kang K-W, Chin JY, et al. Major determinant of the occurrence of pacing-induced cardiomyopathy in complete atrioventricular block: a multicentre, retrospective analysis over a 15-year period in South Korea. BMJ Open 2018;8:e19048.

20 Khurshid S, Obeng-Gyimah E, Supple GE, et al. Reversal of pacinginduced cardiomyopathy following cardiac resynchronization therapy. JACC Clin Electrophysiol 2018;4:168-77.

21 Birnie DH, Ha A, Higginson L. Impact of QRS morphology and duration on outcomes after cardiac resynchronization therapy. Circulation: Heart Failure 2013;6:1190-8.

22 Cao Z, Jia Y, Zhu B. BNP and NT-proBNP as diagnostic biomarkers for cardiac dysfunction in both clinical and forensic medicine. Int $J$ Mol Sci2019;20:1820.

23 Caraballo $\mathrm{C}$, Desai NR, Mulder $\mathrm{H}$, et al. Clinical implications of the New York heart association classification. J Am Heart Assoc 2019;8:e014240.

24 Zhang J, Guo J, Hou X, et al. Comparison of the effects of selective and non-selective his bundle pacing on cardiac electrical and mechanical synchrony. Europace 2018;20:1010-7.

25 Lewis AJM, Foley P, Whinnett Z, et al. His bundle pacing: a new strategy for physiological ventricular activation. J Am Heart Assoc 2019;8.

26 Vijayaraman P, Cano Óscar, Koruth JS, et al. His-Purkinje conduction system pacing following transcatheter aortic valve replacement. JACC Clin Electrophysiol 2020;6:649-57. 